\title{
Development of the Normal Spline Method for Linear Integro-Differential Equations ${ }^{\star}$
}

Vladimir K. Gorbunov, Vyacheslav V. Petrischev, and Vyacheslav Y. Sviridov

Ulyanovsk State University, L.Tolstoy street 42 , 432970 Ulyanovsk, Russia,

gvk@vens.ru, Vyacheslav.Petrischev@transas.com, meria@mv.ru

\begin{abstract}
The normal spline method is developed for the initial and boundary-value problems for linear integro-differential equations, probably being unresolved with respect to the derivatives, in Sobolev spaces of the arbitrary smoothness. It allows to solve a high-order systems without the reduction to first-order ones. The solving system can be arbitrary degenerate (with high differentiation index or irreducible to normal form). The method of nonuniform collocation grid creation for stiff problems is offered. Results of numerical solution to test problems are demonstrated.
\end{abstract}

\section{Introduction}

The problem under consideration is the system of integro-differential equations

$$
A(t) \dot{x}(t)+B(t) x(t)-\int_{0}^{1} K(t, s) x(s) d s=f(t), \quad 0 \leq t \leq 1,
$$

with conditions

$$
C x(0)+D x(1)=g .
$$

Here $x, f, g \in R^{n}, A(t), B(t), K(t, s), C, D$ are square $n$-order matrices. The function $f(t)$ and the coefficients of matrices are continuous and have so many derivatives as it needs to guarantee appropriate smoothness of the solution $x(t)$ that exists on assumption and belongs to the Hilbert-Sobolev space $W_{2, n}^{l}$ with norm

$$
\|x\|_{l, n}=\left\{\sum_{i=1}^{n}\left[\sum_{r=0}^{l-1}\left(x_{i}^{(r)}(0)\right)^{2}+\int_{0}^{1}\left(x_{i}^{(l)}(s)\right)^{2} d s\right]\right\}^{1 / 2} .
$$

Here and later $x_{i}^{(r)}(t)$ are derivatives of the order $r \geq 0$, also $\dot{x}_{i}=x_{i}^{(1)}$.

The matrices of the system (1) may be arbitrary degenerate. It covers the problem of solving in universal form to differential-algebraic equations (DAEs) with any differentiation index [1] and singular systems having not the finite index (irreducible to normal Cauchy form) [2].

\footnotetext{
* Supported by Russian Foundation for Basic Research, project $N^{o}$ 01-01-00731
} 
There exist special methods [1], 3] for solving to degenerate ordinary differential equations (ODEs) working in the case when the matrix rank of the main part of the solving system is constant.

In [4], 5] there was developed a variational normal spline (NS) method for the problem (1),(2). The cases of infinite intervals $[0, \infty)$ and $(-\infty, \infty)$ were also covered. This method is based on the transfer to the finite collocation system of the equalities (1) and the finding an element of the minimal norm. In singular case the problem (11), (2) may have a set of solutions (affine) and the NS method provides then an approximation of the normal solution denoting by $x^{0}[5]$.

The theoretical basis of the NS method is the classic functional analysis results: the theorem of embedding of Sobolev spaces in the Chebyshev one [6] and Riesz theorem [7] of canonical representation of linear continuous functional in Hilbert spaces as inner product. Here the coordinate system is not a priori given and is generated by the equation coefficients and the space $W_{2, n}^{l}$ topology. This representation is based on using some reproducing kernel $[8]$ defined by the norm (3).

One can find the results of the realization of the NS method for ODEs under $l \in\{1,2\}$ in [4], [5]. The method was realized for arbitrary integer $l$ in [9] where general view of reproducing kernels were created. It allows to solve a high-order systems without their reduction to the first-order ones [10] and to solve problems with a high differentiation index. The NS method also was developed for twodimensional problems of the computational tomography [11].

The main purpose of this work is to produce a computational scheme of the NS method for the systems of integro-differential equations (1) in $W_{2, n}^{l}$ with $l \geq 2$. Also the NS method is developed for stiff problems. The using of nonuniform adaptive grids is appropriate in these problems. The different strategies of the collocation grids improvement are based on the theoretical estimates of the NS method precision. The improvement consists in a serial grid modification (addition and/or moving) with the goal to minimize the norm of the current system discrepancy. We present also results of the numerical solving to degenerate and stiff problems.

\section{The Normal Spline Method}

The NS method is a collocation type one. Introduce some grid

$$
0 \leq t_{1}<t_{2}<\ldots<t_{m} \leq 1
$$

and consider the collocation system

$$
A\left(t_{k}\right) \dot{x}\left(t_{k}\right)+B\left(t_{k}\right) x\left(t_{k}\right)-\int_{0}^{1} K\left(t_{k}, s\right) x(s) d s=f\left(t_{k}\right), \quad k=1, \ldots, m .
$$

For this system we pose a problem of a normal (in norm (3i) ) solution. In the case of the compatible system (11), (2) the solution exists and is uniquely defined. 
This fact is the consequence of the theorem [6] about the embedding of Sobolev space $W_{2, n}^{l}[0,1]$ to the Chebyshev one $C_{n}^{l-1}[0,1]$.

Denote the left parts of the system (5), (2) as

$$
l_{i k}(x)=\left\{\begin{array}{l}
\sum_{j=1}^{n} c_{i j} x_{j}(0)+d_{i j} x_{j}(1), \quad k=0 ; \\
\sum_{j=1}^{n} a_{i j}\left(t_{k}\right) \dot{x}_{j}\left(t_{k}\right)+b_{i j}\left(t_{k}\right) x_{j}\left(t_{k}\right)-\int_{0}^{1} k_{i j}\left(t_{k}, s\right) x(s) d s, 1 \leq k \leq m,
\end{array}\right.
$$

where $1 \leq i \leq n$. Respectively, the system (5), (2) takes the form

$$
l_{i 0}(x)=g_{i}, \quad l_{i k}(x)=f_{i}\left(t_{k}\right), \quad 1 \leq i \leq n, \quad 1 \leq k \leq m .
$$

The functions (6) may be considered as linear continuous functionals in the vector-functions space $W_{2, n}^{l}[0,1]$. Respectively, each equation of the system (7) defines a hyperplane and the system solution set (an intersection of the hyperplanes) will be nonempty, convex and closed. The minimal norm element of this intersection is named the normal spline $x^{m}$. It is known [4], the sequence of the normal splines $x^{m}$ converges to a normal solution $x^{0}$ in the norm (3) when the maximum step of the grid (4) tends to zero.

According to the Riesz theorem [7] a linear continuous functional may be represented as a inner product corresponding to the norm (3), i.e.

$$
l_{i k}(x)=\left\langle h^{\mu(i, k)}, x\right\rangle_{(n)}=\sum_{j=1}^{n}\left\langle h_{j}^{\mu(i, k)}, x_{j}\right\rangle_{(1)} .
$$

Here $\mu(i, k)=n k+i, 1 \leq i \leq n, 0 \leq k \leq m$.

Elements $h_{j}^{m}$ of the representation (8) may be found with the help of the reproducing kernel $G(s, t)$ of the scalar function space $W_{2,1}^{l}[0,1]$. Remind [8], the reproducing kernel is a such function $G(s, t)$ that

1. $G(\cdot, t) \in W_{2,1}^{l}[0,1]$ for any $t \in[0,1]$,

2. $x_{i}(t)=\left\langle G(\cdot, t), x_{i}\right\rangle$ for any $x_{i}(\cdot) \in W_{2,1}^{l}[0,1]$ and any $t \in[0,1]$.

Correspondingly,

$$
h_{j}^{\mu(i, k)}(s)=\left\{\begin{array}{l}
c_{i j} G(s, 0)+d_{i j} G(s, 1), \quad k=0 \\
a_{i j}\left(t_{k}\right) G_{s}^{\prime}\left(s, t_{k}\right)+b_{i j}\left(t_{k}\right) G\left(s, t_{k}\right)- \\
-\int_{0}^{1} k_{i j}\left(t_{k}, \tau\right) G(s, \tau) d \tau, \quad 1 \leq k \leq m .
\end{array}\right.
$$

By virtue of (6) and (8) the system (7) is represented as

$$
\left\langle h^{\mu}, x\right\rangle_{(n)}=\bar{f}_{\mu}, \quad \bar{f}_{\mu}=\left\{\begin{array}{l}
g_{i}, \quad k=0 ; \\
f_{i}\left(t_{k}\right), 1 \leq k \leq m .
\end{array}\right.
$$


According to the generalized Lagrange method the normal solution of the linear equations system (10) can be written in the form

$$
x^{m}(s)=\sum_{\mu=1}^{(m+1) n} u_{\mu} h^{\mu}(s) .
$$

Coefficients $u_{\mu}$ are defined by the system

$$
\sum_{\nu=1}^{(m+1) n} g_{\mu \nu} u_{\nu}=\bar{f}_{\mu}, \quad 1 \leq \mu \leq(m+1) n
$$

where $g_{\mu \nu}$ are the coefficients of the Gram matrix of the system $\left\{h^{\mu}\right\}$, i.e.

$$
g_{\mu \nu}=\left\langle h^{\mu}, h^{\nu}\right\rangle_{(n)}=\sum_{i=1}^{n}\left\langle h_{i}^{\mu}, h_{i}^{\nu}\right\rangle_{(1)}
$$

Thus the realization of the NS method with given decomposition of an interval is reduced to the forming of the Gram matrix coefficients according to (13), the solving to the system of linear equation (12) with symmetric, positive defined (as a rule) matrix $\left\{g_{\mu \nu}\right\}$ and the creation of the solution $x^{m}(s)$ at arbitrary point $s \in[0,1]$ with respect to (9), (11).

In 9] it was shown that the kernel $G(s, t)$ for the norm (3) is a Green function of some boundary-value problem and is given by the formula

$$
G(s, t)=\left\{\begin{array}{l}
\sum_{i=0}^{l-1} \frac{t^{i}}{i !}\left(\frac{s^{i}}{i !}-(-1)^{l+i} \frac{s^{2 l-i-1}}{(2 l-i-1) !}\right), \quad 0 \leq s \leq t \leq 1 \\
G(t, s), \quad 0 \leq t<s \leq 1
\end{array}\right.
$$

\section{The Adaptive Grid Construction}

Define the discrepancy of the equation (1) on the function $x^{m}$ :

$$
\varphi(t)=A(t) \dot{x}^{m}(t)+B(t) x^{m}(t)-\int_{0}^{1} K(t, s) x^{m}(s) d s-f(t) .
$$

In [5] the evaluation

$$
\left\|x^{m}-x^{0}\right\|_{l, n} \leq \hat{c}\|\varphi\|_{(l-1), n}
$$

was obtained. The $\hat{c}$ is constant that is depended only on the system (1), (2) coefficients.

The evaluation (16) opens the way to creation of optimal nonuniform grids under given number of nodes $m$, that is very important for the stiff problem solving. The convergence in norm implies a uniform convergence, therefore in 
the process of the method realization we should proceed to new grids decreasing the value $\|\varphi\|_{(l-1), n}$.

There are equalities $\varphi\left(t_{k}\right)=0$ in the collocation nodes hold hence the minimization of the discrepancy norm may be achieved using a simple and reliable heuristic method: to add nodes into subintervals with the greatest intermediate (between nodes) values of $\varphi(t)$. The detailed algorithm of the step-by-step concentration of the grids was offered in [5]. This adaptive grid is named an adding grid with two parameters: the number of start nodes and the number of added nodes on each step.

Another strategy of creation of the adaptive grid with fixed nodes number is based on minimization of the value $\|\varphi\|_{(l-1), n}$ as a function of nodes with the constrains (4). Denote

$$
\psi_{l-1}\left(t_{1}, \ldots, t_{m}\right)=\left\|\varphi\left(s, t_{1}, \ldots, t_{m}\right)\right\|_{(l-1), n}^{2} .
$$

Here the index $l-1$ corresponds to the normal spline $x^{m} \in W_{2, n}^{l}[0,1]$. The grid (4) providing the minimum of function $\psi_{l-1}\left(t_{1}, \ldots, t_{m}\right)$ is named optimal.

In case, when the system (1), (2) is regular (matrix $A(t)$ is non-degenerate), the smoothness of function $\psi_{l-1}$ is defined by the index of derivative $l$ and the reproducing kernel $G(s, t)$ characteristics. The last has continuous derivatives with order up to $2 l-2$. As it is shown in [12 it provides the differentiability of the function $\psi_{l-1}$ with respect to $t_{k}$ for $l \geq 3$. In this case the appropriate analytic formulas for partial derivatives were obtained. We omit them because of their complexity. In general case one may use some direct method (e.g. HookeJeeves [13]) to minimize the function $\psi_{l-1}$.

We turn our attention to the solving of an initial problem for the differential equation (1) $(K \equiv 0)$, i.e. the condition (2) has the form $x(0)=g$. In this case the most effective NS method realization scheme is the sequential normal spline creation on partial adjoining subintervals with a small number of nodes (up to two). The effectiveness is achieved as a result of reducing of the Gram matrix dimension since the calculation of its elements and solving the linear equation system are the most time-consuming part of the algorithm.

If partial interval is sufficiently small we can use the grid with two nodes and create the spline on two nodes $\left\{t_{k-1}, t_{k}\right\}$ on each step. The initial values on second and following subintervals are defined as final values of the created spline on the previous subinterval.

Define some admissible level $\varepsilon$ of local measure of error evaluation $\psi_{l-1}^{k}\left(t_{k-1}, t_{k}\right)$ defined similar to (17). This value should be estimated by using some quadrature formula.

The simple variant of the algorithm is the next.

1. $k:=1, t_{0}:=0$.

2. $t_{k}:=1$.

3. To construct the NS for the Cauchy problem (1) on $\left(t_{k-1}, t_{k}\right)$.

4. If $\psi_{l-1}^{k}<\varepsilon$ then go to 6 .

5. $t_{k}:=\left(t_{k}+t_{k-1}\right) / 2$, go to 3 . 
6. If $t_{k}=1$, then end else $t_{k-1}:=t_{k}$.

7. $k:=k+1$, go to 2 .

As a result we obtain the grid that provides the given level of the local discrepancy norm value.

The similar sequential algorithm can be applied for solution of integrodifferential equations of Volterra type. In this case it is necessary to make the next modification of algorithm: on step 3 we construct the normal spline for the equation

$$
\begin{gathered}
A(t) \dot{x}(t)+B(t) x(t)-\int_{t_{k-1}}^{t} K(t, s) x(s) d s=f(t)+\int_{0}^{t_{k-1}} K(t, s) x^{m}(s) d s, \\
t_{k-1} \leq t \leq t_{k} .
\end{gathered}
$$

\section{Numerical Implementation}

Example 1. The initial problem from [3] for ODE (11) where $K \equiv 0$ and

$$
A(t)=\left(\begin{array}{ll}
t & 1 \\
0 & 0
\end{array}\right), \quad B(t)=\left(\begin{array}{cc}
\gamma & -\delta \\
t & 1
\end{array}\right) .
$$

The system (1) with condition $\gamma+\delta t \neq 1$ has the differentiation index 2 and the unique solution with any right part $f \in C_{2}^{2}[0,1]$. Besides, it was shown [3] that classical difference Euler, Runge-Kutta and multistep methods diverge or are not applied for $\gamma, \delta$ such that $|\gamma+\delta t|<1$. The NS method gives approximate solution in any compatible case.

Table 1 presents results of the initial problem solving for exact desired function

$$
x(t)=\left(\begin{array}{c}
e^{t} \sin (t) \\
\cos (t)
\end{array}\right)
$$

on interval $t \in[0,1]$ with uniform grid of 51 points. Values of the obtained normal splines deviations from exact solution on doubled grid are shown. The cases of mentioned above condition violation is marked by asterix $(*)$. In these cases the non-uniqueness of the solution is possible. The normal spline approximate the normal solution.

One can see that increasing of the spline smoothness leads to the increasing of the approximation accuracy.

Example 2. Consider the second-order initial problem

$$
\begin{gathered}
p(t) \ddot{x}(t)+q(t) \dot{x}(t)+r(t) x(t)=f(t), \quad 0 \leq t \leq 1, \\
x(0)=g_{1}, \quad \dot{x}(0)=g_{2}
\end{gathered}
$$

where $p(t)=\sin (10 t), q(t)=10 \cos (10 t), r(s)=-1$. The function $f(t)$ corresponds to the solution $x(t)=t e^{-t}+e^{-k t}, k>1$. 
Table 1.

\begin{tabular}{|c|c|c|c|c|c|c|}
\hline \multirow{2}{*}{$\gamma \backslash \delta$} & \multicolumn{2}{|c|}{-1} & \multicolumn{2}{c|}{0} & \multicolumn{2}{c|}{2} \\
\cline { 2 - 7 } & $\mathrm{l}=2$ & $\mathrm{l}=3$ & $\mathrm{l}=2$ & $\mathrm{l}=3$ & $\mathrm{l}=2$ & $\mathrm{l}=3$ \\
\hline 2 & $1.36 \mathrm{e}-1^{*}$ & $4.66 \mathrm{e}-04^{*}$ & $1.83 \mathrm{e}-2$ & $3.43 \mathrm{e}-05$ & $6.02 \mathrm{e}-3$ & $1.34 \mathrm{e}-05$ \\
\hline 0 & $1.09 \mathrm{e}-2$ & $2.74 \mathrm{e}-05$ & $2.09 \mathrm{e}-2$ & $4.72 \mathrm{e}-05$ & $2.06 \mathrm{e}-2{ }^{*}$ & $3.50 \mathrm{e}-05^{*}$ \\
\hline-1 & $7.79 \mathrm{e}-3$ & $1.97 \mathrm{e}-05$ & $1.08 \mathrm{e}-2$ & $2.73 \mathrm{e}-05$ & $9.19 \mathrm{e}-2{ }^{*}$ & $3.45 \mathrm{e}-04^{*}$ \\
\hline-2 & $5.58 \mathrm{e}-3$ & $1.58 \mathrm{e}-05$ & $7.31 \mathrm{e}-3$ & $1.97 \mathrm{e}-05$ & $1.95 \mathrm{e}-2$ & $4.59 \mathrm{e}-05$ \\
\hline
\end{tabular}

The coefficients $p(t), q(t)$ have zero values on the interval $[0,1]$ and the solution is stiff under large $k$. The variable degeneracy of the coefficients makes difficulties for using known difference methods for stiff and ADEs [1].

We use the transfer to integro-differential equation

$$
\begin{aligned}
p(t) \dot{x}(t) & +(q(t)-\dot{p}(t)) x(t)-\int_{0}^{t}(\dot{q}(s)-\ddot{p}(s)-r(s)) x(s) d s= \\
& =\int_{0}^{t} f(s) d s+q(0) x(0)+p(0) \dot{x}(0)-\dot{p}(0) x(0) .
\end{aligned}
$$

The effectiveness of this way in stiff cases was shown in [5].

Table 2 presents some results for two variants of the NS method: two-nodes sequential scheme and uniform grid one. The problem was solved in the space $W_{2}^{2}$. Note, that value $\varepsilon$ is used in the sequential scheme and it defines the obtained number of nodes.

Table 2.

\begin{tabular}{|c|c|c|c|c|}
\hline $\mathrm{k}$ & $\varepsilon$ & $\mathrm{m}$ & sequential & uniform \\
\hline 100 & $1.00 \mathrm{e}-3$ & 24 & $5.64 \mathrm{e}-02$ & $1.07 \mathrm{e}+00$ \\
\hline$-/ /-$ & $1.00 \mathrm{e}-5$ & 103 & $7.69 \mathrm{e}-03$ & $2.14 \mathrm{e}-01$ \\
\hline 1000 & $1.00 \mathrm{e}-5$ & 85 & $1.61 \mathrm{e}-02$ & $9.30 \mathrm{e}-01$ \\
\hline
\end{tabular}

\section{Conclusion}

Presented above numerical results also as results of [4], [5], 9], 10], [12] demonstrate the ability of the NS method to obtain appropriate approximation of solutions to arbitrary degenerate problems of linear differential and integrodifferential equations. Particularly for equations that can't be reduced to the normal Cauchy form, i.e. equations not having of a differentiation finite index. We know only theoretical works devoted to such kind of ODEs problems [2]. We are inclined to explain the success of the NS method by using in its base of the Sobolev spaces theory that is outstanding achievement of functional analysis. 


\section{References}

1. Hairer, E., Wanner, G.: Solving Ordinary Differential equations II. Stiff and Differential-algebraic Problems. Springer-Verlag, Berlin (1996)

2. März, R., Weinmüller, E.B.: Solvability of boundary value problems for systems of singular differential-algebraic equations. SIAM J. Math. Anal. Vol. 24.1 (1993) 200-215

3. Bulatov, M.V., Chistyakov, V.F.: About the numerical method of differantialalgebraic equations solving. Comput. Math. Math. Phys. Vol. 42.4 (2002) 459-470

4. Gorbunov, V.K.: The method of normal spline-collocation. Comput. Math. Math. Phys. Vol. 29. 2 (1989) 212-224

5. Gorbunov, V.K.: Ekstremalnie zadachi obrabotki rezul'tatov izmerenei. Ilim, Frunze (1990)

6. Sobolev, S.L.: Applications of functional analysis to mathematical physics. Amer. Math. Soc. Providence RI (1963)

7. Balakrishnan, A.: Applied Functional Analysis. Springer-Verlag, New York (1976)

8. Aronszajn, N.: Theory of reproducing kernels. Tranzactions of the AMS 68 (1950) 337-404

9. Gorbunov, V.K., Petrischev, V.V.: Developing of the normal spline-collocation method for linear differential equations. Comput. Math. Math. Phys. (2003) (to appear)

10. Gorobetz, A.S.: Metod normal'nih splainov dlya system ODU vtorogo poryadka i zadach matematicheskoi phiziki. Differencial'nie uravneniya i ih prilogeniya: Sbornik trudov mejdunarodnoy konferencii. Samara (2002) 99-104

11. Kohanovsky, I.I.: Normalnije splaini v vichislitel'noi tomografii. Autometria 2 (1995) 84-89

12. Sviridov, V. Yu.: Optimizaciya setok metoda normal'nih splainov dlya integrodifferencial'nih uravnenei. Trudi Srednevolgskogo matematicheskogo obschestva. SVMO, Saransk 3-4 (2002) 236-245

13. Himmelblau, D.M.: Applied nonlinear programming. McGraw-Hill Book Company, Texas (1972) 